M. ITOH

KODAI MATH. J.

23 (2000), 358-375

\title{
MINIMALLY IMMERSED LEGENDRIAN SURFACES IN SASAKIAN 5-MANIFOLDS
}

\author{
MitsUHIRO ITOH
}

\begin{abstract}
Minımal, Legendrian surfaces in a Sasakıan 5-manifold are consıdered in terms of the cubic differential form and a generalization of the theorem given by S. Yamaguch et al is obtained. A lower bound for the index of second variation for those surfaces is also derived.
\end{abstract}

\section{§1. Introduction}

The purpose of this paper is to investigate Legendrian surfaces minimally immersed in a Sasakian 5-manifold.

Totally real or Lagrangian submanifolds of a Kähler manifold are recently well investigated, as is surveyed in [4]. In contrast with this, there is a few knowledge of geometry of Legendrian submanifolds of a Sasakian manifold, although in the regular contact case its situation is parallel to geometry of Lagrangian submanifolds.

We concentrate upon minimal Legendrian surfaces in a 5-dimensional Sasakian manifold and generalize a theorem in [11] given by S. Yamaguchi, $\mathbf{M}$. Kon and Y. Miyahara, held for minimal Legendrian surfaces in a 5-dimensional Sasakian space form, a special kind of Sasakian 5-manifold.

A Sasakian 5-manifold is a metric contact manifold $(M, \eta, \xi, \phi, g)$ for which the $(1,1)$-tensor $\phi$ satisfies

$$
\left(\tilde{\nabla}_{X} \phi\right) Y=g(X, Y) \xi-\eta(Y) X
$$

for the Levi-Civita connection $\tilde{\nabla}$.

A surface in a Sasakian 5-manifold is called Legendrian when its tangent spaces all belong to the contact subbundle $\operatorname{Ker} \eta=\{X \in T M \mid \eta(X)=0\}$ of the tangent bundle $T M$.

The unit 5-sphere $S^{5}$ with a standard metric, a typical Sasakian space form, admits as Legendrian embeddings of Riemann surfaces a totally geodesic em-

1991 Mathematical Subject Classification: 53A10, 53C25

Received July 21, 1999; revised March 9, 2000. 
bedding of a round 2-sphere $S^{2}([2])$ and a minimal embedding of a flat 2-torus $T^{2}([5])$.

However, any compact Riemann surface of negative constant Gaussian curvature $K$ can not be minimally immersed in $S^{5}$ as Legendrian surface, as shown by S. Yamaguchi et al in the following theorem.

THEOREM ([11]). Let $M$ be a 5-dimensional Sasakian space form, i.e., a Sasakian 5-manifold with constant $\phi$-sectional curvature $k$ and $\Sigma \rightarrow M$ be $a$ minimally immersed Legendrian surface in $M$. geodesic.

(i) If $\Sigma$ is a compact Riemann surface of genus $g=0$, then $\Sigma \rightarrow M$ is totally

(ii) If $\Sigma$ is a complete Riemann surface with $K \geq 0$, then $\Sigma \rightarrow M$ is totally geodesic or $\Sigma$ is flat.

(iii) If $\Sigma \rightarrow M$ is a complete Riemann surface with $K \leq 0$ and $(k+3) / 4-$ $K \geq \alpha>0$ for a constant $\alpha$, then $K=0$.

This theorem is shown in [11] by making use of a cubic differential $2=$ $2(z) d z^{3}$ on the surface, which is induced from the second fundamental form of the immersion.

However their proof relies on the Sasakian space form condition. We can relax this constant $\phi$-sectional curvature condition from their theorem by investigating explicitly the holomorphicity of the cubic differential.

In order to relax the Sasakian space form condition we employ in terms of isothermal coordinate the curvature hypothesis

$$
g\left(\tilde{R}\left(\frac{\partial}{\partial \bar{z}}, \frac{\partial}{\partial z}\right) \frac{\partial}{\partial z}, \phi\left(\frac{\partial}{\partial z}\right)\right)=0
$$

with respect to the Riemannian curvature tensor $\tilde{R}$ of $(M, g)$.

The holomorphicity of $\mathscr{Q}$ is guaranteed by this hypothesis even though $M$ is not a Sasakian space form.

We then have our main theorems.

THEOREM A. Let $\Sigma \rightarrow M$ be a compact, connected orientable Legendrian surface minimally immersed in a Sasakıan 5-manifold.

Assume $\Sigma \rightarrow M$ satisfies the curvature hypothesis (2).

(i) If $\Sigma$ has genus zero, then the immersion $\Sigma \rightarrow M$ is totally geodesic.

(ii) If the Gaussian curvature $K \geq 0$, then either $\Sigma \rightarrow M$ is totally geodesic or $(\Sigma, g)$ is flat and the second fundamental form $\sigma$ has constant norm.

(iii) If $K \leq 0$ and $M$ has positive sectional curvature, then $(\Sigma, g)$ is flat 2-torus.

THEOREM B. Let $\Sigma \rightarrow M$ be a compact, open connected, orientable Legendrian surface minimally immersed in a Sasakıan 5-manifold.

Assume $\Sigma \rightarrow M$ satisfies the curvature hypothesis (2).

(i) If the Gaussian curvature $K \leq 0$ and there is a constant $\kappa$ such that $\tilde{K} \geq$ $\kappa>0$ for the sectional curvature $\tilde{K}$ of $(M, g)$, then $(\Sigma, g)$ is flat. 
(ii) If the Gaussian curvature $K \geq 0$ and the sectional curvature $\tilde{K}$ of $M$ is uniformly bounded from above i.e., $\tilde{K}<c$ for a constant $c$, then either the immersion $\Sigma \rightarrow M$ is totally geodesic, or $(\Sigma, g)$ is flat and the second fundamental form $\sigma$ has constant norm and the covariant derivative $\nabla^{\prime} \sigma$ has only $\xi$-component.

Theorems A, B generalize the theorems in [11]. In fact, if a Sasakian 5manifold $M$ is Sasakian space form, then it is seen from the curvature property of the space form that any Legendrian surface is curvature invariant, in other words

$$
\tilde{R}(T \Sigma, T \Sigma) T \Sigma \subset T \Sigma
$$

so that the hypothesis (2) is fulfilled, since $g(T \Sigma, \phi(T \Sigma))=0$.

We give several remarks relating to our theorems.

Remarks. (i) Theorem A, (iii) holds even if $\Sigma \rightarrow M$ does not satisfy (2).

(ii) In the above theorems the dimension of $M$ can be relaxed into $2 n+1 \geq$ 5 , provided a minimally immersed surface $\Sigma \rightarrow M$ fulfills

$$
\begin{aligned}
T_{p} \Sigma \oplus \phi\left(T_{p} \Sigma\right) & \subset \operatorname{Ker} \eta=\xi^{\perp}, \\
\sigma\left(T_{p} \Sigma, T_{p} \Sigma\right) & \subset \phi\left(T_{p} \Sigma\right)
\end{aligned}
$$

for any point $p$.

(iii) When a Sasakian manifold $M$ is regular contact, $M$ has a BoothbyWang fibration over a Hodge manifold $\check{M}$ with a circle fibre and admits a Riemannian submersion $\pi: M \rightarrow \check{M}$ ([3], [6]). As shown by Rechziegel in [9] a minimal Legendrian immersion $f: \Sigma \rightarrow M$ corresponds in a local sense to a minimal Lagrangian immersion $\pi \circ f: \Sigma \rightarrow \check{M}$.

The Boothby-Wang fibration of the Sasakian 5-manifold $S^{5}$ is the Hopf fibration $S^{5} \rightarrow C P^{2}$ so that the typical minimally embedded Legendrian surfaces $S^{2} \subset S^{5}$ and $T^{2} \subset S^{5}$ correspond to the minimally embedded Lagrangian surfaces $\boldsymbol{R} P^{2} \subset C P^{2}$ and $T^{2} / Z_{3} \subset C P^{2}$ where $Z_{3}$ is the cyclic group of order 3 .

(iv) M. Kameda obtained quite similar results relating to Legendrian submanifolds of $\eta$-parallel mean curvature in a Sasakian manifold (see [7]).

In the final section we will deal with the index of a minimal Legendrian surface in a Sasakian 5-manifold with respect to the second variation of area and will obtain in Proposition 6.2 a formula of the index form in terms of intrinsic geometry of $M$ and a surface $\Sigma$. As a consequence we get Theorem 6.1 in which the index has a lower bound in terms of the space of holomorphic vector fields on $\Sigma$. Similar results are obtained in [8] for minimal surfaces in a complex 2dimensional Kähler manifold.

The author is grateful to the referee for valuable comments.

\section{§2. Sasakian manifolds and Legendrian surfaces}

A contact manifold $(M, \eta)$ of dimension $2 n+1$ is called metric contact, when it admits a Riemannian metric $g$ and a tensor $\phi \in \Gamma(M, \operatorname{End}(T M))$ sat- 
isfying

$$
\begin{gathered}
\phi(\phi X)=-X+\eta(X) \xi \\
g(\phi(X), \phi(Y))=g(X, Y)-\eta(X) \eta(Y), \\
\frac{1}{2} d \eta(X, Y)=g(X, \phi(Y)) .
\end{gathered}
$$

Here $\xi$ is the Reeb field, that is, $\xi$ is a vector field on $M$ satisfying $\eta(\xi)=1$ and $d \eta(\xi, X)=0$ for any vector $X$.

A metric contact manifold is called Sasakian if

$$
\left(\tilde{\nabla}_{X} \phi\right)(Y)=g(X, Y) \xi-\eta(Y) X .
$$

Remark that $\phi$ restricted to the contact subbundle $\operatorname{Ker} \eta$ is from (5) an almost complex structure. The Reeb field $\xi$ is normal to $\operatorname{Ker} \eta$ and fulfills

$$
\phi(\xi)=0, \quad \tilde{\nabla}_{X} \xi=-\phi(X) .
$$

which shows that $\xi$ gives a unit Killing field over $M$.

Like a holomorphic sectional curvature of a Kähler manifold, a sectional curvature of a $\phi$-invariant plane section in Ker $\eta$ of a Sasakian manifold is defined and is called a $\phi$-sectional curvature. A Sasakian manifold of constant $\phi$-sectional curvature is called a Sasakian space form.

Typical examples of Sasakian space form are an odd dimensional unit sphere $S^{2 n+1} \subset C^{n+1}$ with the characteristic field $\xi=J(\partial / \partial r), \boldsymbol{R}^{2 n+1}$ with the standard contact structure and the product space $B^{n} \times \boldsymbol{R}$ with a contact form $\eta=\omega+d t$ where $B^{n}$ is a simply connected bounded domain in $C^{n}$ with the Kähler form $d \omega$.

We assume $(M, \eta, \xi, \phi, g)$ be a Sasakian 5-manifold.

Let $\Sigma \rightarrow M$ be a connected immersed surface in $M$.

Then the induced tangent bundle of $M$ splits as

$$
\left.T M\right|_{\Sigma}=T \Sigma \oplus N \Sigma
$$

where $N \Sigma$ is the normal bundle of the immersion. Then the Gauss formula and the Weingarten formula are

$$
\begin{gathered}
\tilde{\nabla}_{X} Y=\nabla_{X} Y+\sigma(X, Y), \\
\tilde{\nabla}_{X} v=-A^{v}(X)+\nabla_{X}^{\perp} v
\end{gathered}
$$

for tangent vectors $X, Y$ of $\Sigma$ and a normal field $v$. Here $\nabla$ is the induced LeviCivita connection on $\Sigma$ and $\sigma$ is the second fundamental form and, $A^{v}$ and $\nabla^{\perp}$ are the shape operator and the normal bundle connection, respectively.

The induced metric on $\Sigma$ is denoted by the same letter $g$ which we abbreviate frequently as $\langle\cdot, \cdot\rangle$.

The equations of Gauss and Codazzi are for tangent vectors $X, Y, V, W$

$$
\begin{aligned}
&\langle R(X, Y) V, W\rangle=\langle\tilde{R}(X, Y) V, W\rangle+\langle\sigma(X, W), \sigma(Y, V)\rangle \\
&-\langle\sigma(X, V), \sigma(Y, W)\rangle, \\
&(\tilde{R}(X, Y) V)^{\perp}=\left(\nabla_{X}^{\prime} \sigma\right)(Y, V)-\left(\nabla_{Y}^{\prime} \sigma\right)(X, V)
\end{aligned}
$$


where $\nabla^{\prime}$ is the covariant differentiation on the bundle $N \Sigma \otimes S\left(T^{*} \Sigma\right)$ given by

$$
\left(\nabla_{X}^{\prime} \sigma\right)(Y, V)=\nabla_{X}^{\perp}(\sigma(Y, V))-\sigma\left(\nabla_{X} Y, V\right)-\sigma\left(Y, \nabla_{X} V\right)
$$

Definition 2.1. An immersed surface $\Sigma \rightarrow M$ is called Legendrian, if $T_{p} \Sigma \subset$ $(\operatorname{Ker} \eta)_{p}$ for any point $p$ of $\Sigma$.

Then, for a Legendrian surface the restricted metric $\left.g\right|_{\operatorname{Ker} \eta}$ is $\phi$-invariant, $\langle\phi(X), \phi(Y)\rangle=\langle X, Y\rangle$ so that at any point it holds

$$
\begin{aligned}
T_{p} \Sigma & \perp \phi\left(T_{p} \Sigma\right) \\
(\operatorname{Ker} \eta)_{p} & =T_{p} \Sigma \oplus \phi\left(T_{p} \Sigma\right),
\end{aligned}
$$

and hence

$$
N \Sigma=\phi(T \Sigma) \oplus \boldsymbol{R} \xi .
$$

In what follows we assume that an immersed surface be Legendrian.

LEMMA 2.1. For a Legendrian surface $\Sigma \rightarrow M$

(i)

$$
\nabla_{X}^{\perp}(\phi(V))=\phi\left(\nabla_{X} V\right)+\langle X, V\rangle \xi
$$

(ii)

$$
\langle\sigma(X, Y), \xi\rangle=0
$$

and

(iii) the 3-tensor defined by

$$
Q(X, Y, W)=\langle\sigma(X, Y), \phi(W)\rangle
$$

is symmetric.

The proof is shown by using the formula (8) together with the fact that a surface is Legendrian.

We assume now that $\Sigma$ is orientable and fix an orientation.

We take an orthonormal frame $\left\{e_{1}, e_{2}\right\}$ of $\Sigma$ compatible with the orientation. Then $\left\{\phi\left(e_{1}\right), \phi\left(e_{2}\right), \xi\right\}$ gives an orthonormal frame of the normal bundle $N \Sigma$ in which $\sigma$ has the components

$$
\sigma_{i j}^{k}=\left\langle\sigma\left(e_{i}, e_{j}\right), \phi\left(e_{k}\right)\right\rangle, \quad i, j, k=1,2
$$

enjoying the symmetry

$$
\sigma_{i j}^{k}=\sigma_{j i}^{k}=\sigma_{i k}^{j}, \quad i, j, k=1,2
$$

The mean curvature vector $H$ is defined by $H=(1 / 2) \sum_{l=1}^{2} \sigma\left(e_{i}, e_{i}\right)$.

Notice that the vector $\phi(H)$ is tangent to $\Sigma$ with norm $|\phi(H)|=|H|$.

Then, in virtue of Hopf's theorem we observe that if $\Sigma$ is a compact, connected orientable Legendrian surface of genus $g(\Sigma) \neq 1$ immersed in a Sasakian 
5-manifold $M$ and have constant norm $|H|$, then $H=0$, in other words, the immersion is minimal.

Associated to the orthonormal frame $\left\{e_{1}, e_{2}\right\}$ compatible with the orientation of $\Sigma$ we define an almost complex structure $J: T \Sigma \rightarrow T \Sigma$ at each point by $J\left(e_{1}\right)=e_{2}, J\left(e_{2}\right)=-e_{1}$. Then $\langle J X, J Y\rangle=\langle X, Y\rangle$ for tangent vectors $X, Y$.

Moreover it holds $\nabla J=0$.

In fact, if we set the connection forms $\omega_{1}^{2}, \omega_{2}^{1}=-\omega_{1}^{2}$ such that for $i=$ $1,2 \nabla_{X} e_{i}=\omega_{i}^{J}(X) e_{j}$ where $j \neq i$, then $\left(\nabla_{X} J\right)\left(e_{1}\right)=\nabla_{X}\left(J e_{1}\right)-J\left(\nabla_{X} e_{1}\right)$ reduces to $\omega_{2}^{1}(X) e_{1}+\omega_{1}^{2}(X) e_{1}=0$ and similarly $\left(\nabla_{X} J\right)\left(e_{2}\right)=0$. So, $J$ is parallel.

Suppose that the mean curvature vector $H$ be a non-zero over a surface $\Sigma$. Then $\phi(H)$ is non-zero tangent vector field over $\Sigma$ so that $\{\phi(H), J(\phi(H))\}$ constitutes a globally defined orthonormal frame field. We have then

Proposition 2.1. Let $\Sigma \rightarrow M$ be a connected orientable, immersed Legendrian surface in a Sasakian 5-manifold. If $H$ is non-zero, and parallel, i.e., $\nabla^{\perp} H=0$, then $\Sigma$ must be flat.

Remark 2.1. The surface $\Sigma$ stated in Proposition 2.1 has genus zero, provided $\Sigma$ is compact.

Proof. We have

$$
\begin{aligned}
\tilde{\nabla}_{X}(\phi(H)) & =\phi\left(\nabla_{X}^{\perp} H+A^{H}(X)\right)+\langle X, H\rangle \xi-\langle\xi, H\rangle X \\
& =\phi\left(A^{H}(X)\right)
\end{aligned}
$$

We take the tangential component to have $\nabla_{X}(\phi(H))=0$ which shows that the tangent vector $\phi(H)$ is parallel.

Q.E.D.

We introduce isothermal coordinates $x, y$ so that $\Sigma$ is a Riemann surface having complex coordinates $z=x+i y$. We define as usual complex vector fields $\partial / \partial z, \partial / \partial \bar{z}$ and their dual $d z, d \bar{z}$.

So the metric has the form

$$
g=E\left(d x^{2}+d y^{2}\right)=\frac{E}{2}(d z \otimes d \bar{z}+d \bar{z} \otimes d z)
$$

and

$$
\left\{e_{1}=\frac{1}{\sqrt{E}} \frac{\partial}{\partial x}, e_{2}=\frac{1}{\sqrt{E}} \frac{\partial}{\partial y}\right\}
$$

is an orthonormal frame compatible with the orientation. The Levi-Civita connection satisfies

$$
\nabla_{\partial / \partial z} \frac{\partial}{\partial \bar{z}}=\nabla_{\partial / \partial z} \frac{\partial}{\partial z}=0, \quad \nabla_{\partial / \partial z} \frac{\partial}{\partial z}=\frac{\partial \log E}{\partial z} \frac{\partial}{\partial z}
$$


In terms of an isothermal coordinate the Gaussian curvature $K$ is written as

$$
K=\frac{1}{2} \Delta_{g} \log E
$$

where $\Delta_{g}=-1 / E\left\{\partial^{2} / \partial x^{2}+\partial^{2} / \partial y^{2}\right\}=-4 / E\left(\partial^{2} / \partial z \partial \bar{z}\right)$ is the Laplacian.

In order to investigate Legendrian surfaces more adequately we take the complexified vector bundles $T \Sigma^{C}$ and $\phi\left(T \Sigma^{C}\right)$ to extend complex linearly the metric $g=\langle\cdot, \cdot\rangle$, the second fundamental form $\sigma$ and $\phi$.

So,

$$
\left\langle\frac{\partial}{\partial z}, \frac{\partial}{\partial z}\right\rangle=\left\langle\frac{\partial}{\partial \bar{z}}, \frac{\partial}{\partial \bar{z}}\right\rangle=0, \quad\left\langle\frac{\partial}{\partial z}, \frac{\partial}{\partial \bar{z}}\right\rangle=\frac{E}{2}
$$

and

$$
\sigma\left(\frac{\partial}{\partial z}, \frac{\partial}{\partial \bar{z}}\right)=\frac{E}{2} H
$$

\section{§3. Minimal Legendrian surfaces and the cubic differential}

Let $\Sigma \rightarrow M$ be a connected, orientable Legendrian surface immersed in a Sasakian 5-manifold which is minimal, i.e., $H=0$.

We give a precise defintion of the cubic differential $\mathscr{Q}=\mathscr{Q}(z) d z^{3}$, a smooth section of the holomorphic complex line bundle $\otimes{ }^{3} \mathscr{T}^{*}\left(\mathscr{T}^{*}\right.$ denotes the bundle of $(1,0)$-forms) over $\Sigma$, by

$$
\mathscr{Q}(z)=\left\langle\sigma\left(\frac{\partial}{\partial z}, \frac{\partial}{\partial z}\right), \phi\left(\frac{\partial}{\partial z}\right)\right\rangle .
$$

LEMMA 3.1. For a minimally immersed Legendrian surface $\Sigma \rightarrow M$

$$
2\|\mathscr{Q}\|^{2}=\|\sigma\|^{2}
$$

and

(ii)

$$
\|\mathscr{Q}\|^{2}(z)=\tilde{K}_{p}-K_{p}, \quad p \in \Sigma
$$

where $z$ is the complex coordinate of a point $p$, and $\tilde{K}_{p}$ and $K_{p}$ denote the sectional curvature of the plane section $T_{p} \Sigma$ and the Gaussian curvature of $\Sigma$, respectively.

Proof. (i) Since the immersion is minimal and Legendrian, $\|\sigma\|^{2}=$ $\sum_{l, j, k=1}^{2}\left(\sigma_{i j}^{k}\right)^{2}$ is

$$
\|\sigma\|^{2}=4\left\{\left(\sigma_{11}^{1}\right)^{2}+\left(\sigma_{11}^{2}\right)^{2}\right\} .
$$

On the other hand $\|\mathscr{Q}\|^{2}$ is measured by the Hermitian fibre metric of $\otimes^{3} \mathscr{T}^{*}$ induced from the metric $g$ so that $\left\langle d z^{3}, d z^{3}\right\rangle=(2 / E)^{3}$ and hence $\|\mathscr{Q}\|^{2}=$ $(2 / E)^{3}|\mathscr{Q}(z)|^{2}$. Here $\mathscr{Q}(z)=E \sqrt{E} / 2\left(\sigma_{11}^{1}-i \sigma_{11}^{2}\right)$. Thus we have (28).

(ii) follows directly from the equation of Gauss.

Q.E.D. 
Proposition 3.1. Let $M$ be a Sasakian 5-manifold of positive sectional curvature and $\Sigma \rightarrow M$ be a compact connected, orientable Legendrian surface immersed minimally in $M$.

(i) If the Gaussian curvature $K \leq 0$, then $(\Sigma, g)$ is a flat 2-torus.

(ii) If $K$ is constant, then either $K=0$ or $K$ is positive constant.

Theorem A, (iii) follows from Proposition 3.1.

Proof. (i) Since $\tilde{K}-K>0$ over $\Sigma$ from the curvature assumption, 2 vanishes nowhere from (29). Hence the bundle $\otimes^{3} \mathscr{T}^{*}$ is trivial as a smooth product bundle so that the first Chern class $3 c_{1}\left(\mathscr{T}^{*}\right)=0$ and thus the genus $g(\Sigma)=1$, namely $\Sigma$ is a 2-torus. That $K=0$ is shown by the Gauss-Bonnet theorem.

(ii) is derived from (i).

Q.E.D.

Remark 3.1. The second fundamental form for a Legendrian surface $\Sigma$ is in general written

$$
\sigma\left(\frac{\partial}{\partial z}, \frac{\partial}{\partial z}\right)=\mathscr{U}(z) \phi\left(\frac{\partial}{\partial z}\right)+\frac{2}{E} \mathscr{2}(z) \phi\left(\frac{\partial}{\partial \bar{z}}\right)
$$

where $\mathscr{U}=\mathscr{U}(z) d z$ is a first order differential over $\Sigma$ given by $\mathscr{U}(z)=$ $\langle H, \phi(\partial / \partial z)\rangle$.

So, if the surface $\Sigma$ is minimally immersed,

$$
\sigma\left(\frac{\partial}{\partial z}, \frac{\partial}{\partial z}\right)=\frac{2}{E} \mathscr{2}(z) \phi\left(\frac{\partial}{\partial \bar{z}}\right)
$$

Moreover, a fourth order differential $\mathscr{W}=\mathscr{W}(z) d z^{4}$ is defined by

$$
\mathscr{W}(z)=\left\langle\sigma\left(\frac{\partial}{\partial z}, \frac{\partial}{\partial z}\right), \sigma\left(\frac{\partial}{\partial z}, \frac{\partial}{\partial z}\right)\right\rangle
$$

and it holds

$$
\mathscr{W}=2 \mathscr{2} \otimes \mathscr{U}
$$

So, $\mathscr{W}$ vanishes identically, provided the immersion is minimal.

LEMMA 3.2 .

$$
\frac{\partial \mathscr{Q}(z)}{\partial \bar{z}}=\left\langle\tilde{R}\left(\frac{\partial}{\partial \bar{z}}, \frac{\partial}{\partial z}\right) \frac{\partial}{\partial z}, \phi\left(\frac{\partial}{\partial z}\right)\right\rangle
$$

Proof. We denote, for simplicity, $\partial / \partial z$ by $Z$. First we show

$$
\frac{\partial}{\partial \bar{z}} \mathscr{2}(z)=\left\langle\nabla_{\bar{Z}}^{\perp}(\sigma(Z, Z)), \phi(Z)\right\rangle .
$$


From the definition of $\mathscr{Q}(z)$ we have in fact

$$
\frac{\partial}{\partial \bar{z}} \mathscr{Q}(z)=\left\langle\nabla_{\bar{Z}}^{\perp}(\sigma(Z, Z), \phi(Z)\rangle+\left\langle\sigma(Z, Z), \nabla_{\bar{Z}}^{\perp}(\phi(Z))\right\rangle\right.
$$

of which the second term vanishes, namely

$$
\left\langle\sigma(Z, Z), \nabla_{\bar{Z}}^{\perp}(\phi(Z))\right\rangle=0 \text {. }
$$

This will be shown as follows.

Since $M$ is Sasakian and the surface is Legendrian, we have from (8)

$$
\nabla_{\bar{Z}}^{\perp}(\phi(Z))=\phi\left(\nabla_{\bar{Z}} Z\right)+\langle\bar{Z}, Z\rangle \xi=\langle\bar{Z}, Z\rangle \xi .
$$

Since $\sigma$ has no $\xi$-component, the term (37) reduces to zero so that

Here we used (23).

$$
\frac{\partial \mathscr{Q}(z)}{\partial \bar{z}}=\left\langle\left(\nabla_{\bar{Z}}^{\prime} \sigma\right)(Z, Z), \phi(Z)\right\rangle \text {. }
$$

In the equation of Codazzi

$$
(\tilde{R}(X, Y) V)^{\perp}=\left(\nabla_{X}^{\prime} \sigma\right)(Y, V)-\left(\nabla_{Y}^{\prime} \sigma\right)(X, V),
$$

we set $Y=V=Z$ and also $X=\bar{Z}$ to have

$$
\left(\nabla_{\bar{Z}}^{\prime} \sigma\right)(Z, Z)=(\tilde{R}(\bar{Z}, Z) Z)^{\perp}+\left(\nabla_{Z}^{\prime} \sigma\right)(\bar{Z}, Z)
$$

whose second term vanishes, because from (23) and (26)

$$
\left(\nabla_{Z}^{\prime} \sigma\right)(\bar{Z}, Z)=\nabla_{Z}^{\perp}(\sigma(\bar{Z}, Z))-\sigma\left(\nabla_{Z} \bar{Z}, Z\right)-\sigma\left(\bar{Z}, \nabla_{Z} Z\right)
$$

reduces to zero. So, we get Lemma 3.2.

Q.E.D.

Thus we get the following proposition which gives the holomorphicity criterion for 2 .

Proposition 3.2. Let $\Sigma \rightarrow M$ be a connected, orientable surface mimimally immersed in a Sasakian 5-manifold.

If the immersion is Legendrian and satisfies the curvature hypothesis (2), then the cubic differential $\mathscr{Q}$ is a holomorphic section of $\otimes^{3} \mathscr{T}^{*}$.

From Proposition 3.2 the following genus theorem is easily derived.

THEOREM 3.1. Let $M$ be a Sasakian 5-manifold and $\Sigma \rightarrow M$ a compact connected, orientable surface immersed in $M$ as a Legendrian submanifold.

Assume that the immersion is minimal and satisfies the curvature hypothesis (2).

(i) If the genus $g(\Sigma)=0$, then $\mathscr{Q}=0$ so that the immersion is totally geodesic.

(ii) If $g(\Sigma)=1$, then $\mathscr{Q}$ vanishes nowhere, provided $\mathscr{Q} \neq 0$ somewhere.

(iii) If $g(\Sigma) \geq 2$, the zero-point set of $\mathscr{Q}$ is non-empty and consists of totally geodesic points, i.e., points where $\sigma=0$.

Theorem 3.1 yields Theorem A, (i). 
Proof. The proof is shown as follows. From the assumptions and the curvature hypothesis $\mathscr{Z}$ is a holomorphic section of $\otimes^{3} \mathscr{T}^{*}$. For a compact Riemann surface $\Sigma$ the dimension $\operatorname{dim} H^{0}\left(\Sigma ; \otimes^{3} \mathscr{T}^{*}\right)=0,1$ or positive according to $g(\Sigma)=0,1$ or $g>1$.

So we see that if $g(\Sigma)=0$, then $\mathscr{Q}=0$ and hence from Lemma 3.1, (i) $\sigma=0$. derived.

When $g(\Sigma)=1$, the bundle $\otimes^{3} \mathscr{T}^{*}$ is a product bundle and hence (ii) is

For the case $g(\Sigma) \geq 2$ it is seen that $\mathscr{Q}$ vanishes somewhere. Otherwise $\mathscr{Q}$ gives a global holomorphic frame to $\otimes^{3} \mathscr{T}^{*}$ and so the genus must be one. This is a contradiction. From Lemma 3.1, (i) the zero-point set $\{p \in \Sigma ; \mathscr{Q}=0\}$ is exactly the set of totally geodesic points.

Q.E.D.

Remark 3.2. With respect to Theorem 3.1, (iii) the number of zero-points of 2 can be represented as

$$
\sum_{l} m_{i}=12(g(\Sigma)-1)=-6 \chi(\Sigma) .
$$

where $\left\{m_{1}, \ldots, m_{\ell}\right\}$ are the multiplicities of the zero-points $\left\{p_{1}, \ldots, p_{\ell}\right\}$ of $\mathscr{Q}$.

Before proceeding to $\S 4$ we give in the following proposition a certain curvature condition equivalent to the curvature hypothesis (2).

Proposition 3.3. For a Legendrian surface $\Sigma \rightarrow M$ immersed in a Sasakian 5-manifold the following are equivalent;

(i) the curvature hypothesis (2) holds,

(ii) there exist a 2-form $\omega$ and a covariant 3-tensor $\alpha$ over $\Sigma$ such that the normal bundle part of the ambient space curvature tensor $\tilde{R}$ satisfies

$$
(\tilde{R}(X, Y) V)^{\perp}=\omega(X, Y) \phi(V)+\alpha(X, Y, V) \xi
$$

for tangent vectors $X, Y, V$ of $\Sigma$.

Proof. (ii) $\Rightarrow(\mathrm{i})$ is easily shown, since $\phi(\partial / \partial z)$ is a normal vector so that the LHS of the curvature hypothesis (2) reduces to $\omega(X, Y)\langle\phi(\partial / \partial z), \phi(\partial / \partial z)\rangle+$ $\alpha(X, Y, V)\langle\xi, \phi(\partial / \partial z)\rangle$ where $Y=V=\partial / \partial z$ and $X=\bar{Y}$, and this vanishes because

$$
\left\langle\phi\left(\frac{\partial}{\partial z}\right), \phi\left(\frac{\partial}{\partial z}\right)\right\rangle=\left\langle\frac{\partial}{\partial z}, \frac{\partial}{\partial z}\right\rangle=0 .
$$

(i) $\Rightarrow$ (ii): Put in the hypothesis (2)

$$
\frac{\partial}{\partial z}=\frac{1}{2} \sqrt{E}\left(e_{1}-\imath e_{2}\right), \quad \frac{\partial}{\partial \bar{z}}=\frac{1}{2} \sqrt{E}\left(e_{1}+i e_{2}\right) .
$$

Then, (i) is equivalent to

$$
\left\langle\tilde{R}\left(e_{1}, e_{2}\right) e_{1}, \phi\left(e_{1}\right)\right\rangle=\left\langle\tilde{R}\left(e_{1}, e_{2}\right) e_{2}, \phi\left(e_{2}\right)\right\rangle
$$


and

$$
\left\langle\tilde{R}\left(e_{1}, e_{2}\right) e_{1}, \phi\left(e_{2}\right)\right\rangle+\left\langle\tilde{R}\left(e_{1}, e_{2}\right) e_{2}, \phi\left(e_{1}\right)\right\rangle=0 .
$$

Since $\Sigma$ is Legendrian, each tangent vector to $\Sigma$ belongs to $\operatorname{Ker} \eta=\xi^{\perp}$. So from formula a) in Lemma, 1 Chapter V, [1]

$$
\left\langle\tilde{R}\left(e_{1}, e_{2}\right) e_{2}, \phi\left(e_{1}\right)\right\rangle+\left\langle\tilde{R}\left(e_{1}, e_{2}\right) \phi\left(e_{2}\right), e_{1}\right\rangle=0
$$

and hence one gets

$$
\left\langle\tilde{R}\left(e_{1}, e_{2}\right) e_{2}, \phi\left(e_{1}\right)\right\rangle=\left\langle\tilde{R}\left(e_{1}, e_{2}\right) e_{1}, \phi\left(e_{2}\right)\right\rangle=0 .
$$

Therefore (i) holds if and only if

$$
\begin{aligned}
& \left(\tilde{R}\left(e_{1}, e_{2}\right) e_{1}\right)^{\perp} \equiv c \phi\left(e_{1}\right) \bmod \boldsymbol{R} \xi \\
& \left(\tilde{R}\left(e_{1}, e_{2}\right) e_{2}\right)^{\perp} \equiv c \phi\left(e_{2}\right) \bmod \boldsymbol{R} \xi
\end{aligned}
$$

for a constant $c$.

This can be written as

$$
\left(\tilde{R}\left(e_{1}, e_{2}\right) V\right)^{\perp}=c \phi(V)+f(V) \xi, \forall V
$$

for some linear form $f=f(V)$ in $V$. (ii).

It is not difficult to see that this is equivalent to the form stated in Q.E.D.

\section{§4. Complete minimal Legendrian surfaces}

Now we assume the surface $\Sigma$ is complete and open.

We have then the following flatness theorem.

Theorem 4.1 (Theorem B, (i) in $\S 1$ ). Let $M$ be a Sasakian 5-manifold with sectional curvature $\tilde{K} \geq \kappa>0$ for a positive constant $\kappa$.

Let $\Sigma \rightarrow M$ be a complete open, orientable Legendrian surface minimally immersed in $M$.

Suppose the surface $\Sigma$ satisfies the curvature hypothesis (2).

If the Gaussian curvature $K$ is nonpositive, then $K$ must be zero everywhere so that $(\Sigma, g)$ is flat.

Proof. 2 is holomorphic from the curvature hypothesis. On the other hand from Lemma 3.1, (ii) $\|\mathscr{2}\|$ is positive over $\Sigma$.

Consider the real valued function $\log \|\mathscr{Q}\|^{2}$. Since

$$
\log \|\mathscr{Q}\|^{2}=\log \mathscr{Q}(z) \overline{\mathscr{Q}}(z)-3 \log E+3 \log 2,
$$

and $\mathscr{Q}(z)$ is holomorphic, we can take the Laplacian of $\log \|\mathscr{Q}\|^{2}$ to have

$$
\Delta_{g} \log \|\mathscr{Q}\|^{2}=-6 K
$$

or equivalently 


$$
\Delta \log \|\mathscr{Q}\|^{2}=-6 E K
$$

which is nonnegative.

Thus the function $\log \|\mathscr{Q}\|^{2}$ is subharmonic. It is bounded from below, since from the formula (29) $\log \|\mathscr{Q}\|^{2}=\log \{\tilde{K}-K\} \geq \log \kappa$.

We define a conformal metric $g^{*}$ over $\Sigma$ by

$$
g^{*}=|\mathscr{Q}(z)|^{2 / 3}\left((d x)^{2}+(d y)^{2}\right)=\frac{1}{2}\|\mathscr{Q}\|^{2 / 3} g
$$

which has zero Gaussian curvature, because

$$
\frac{1}{2} \Delta_{g^{*}}\left(\frac{1}{3} \log |\mathscr{Q}(z)|^{2}\right)=0 .
$$

Since $g$ is complete and $(1 / 2)\|\mathscr{Q}\|^{2 / 3} \geq(1 / 2) \kappa^{1 / 3}, g^{*}$ is also complete. Hence its universal covering space $\tilde{\Sigma}$ is the Euclidean complex plane over which any subharmonic function which is bounded from below must be constant. Thus, $\log \|\mathscr{Q}\|^{2}$ is constant, when lifted to $\tilde{\Sigma}$ and hence also constant over $\Sigma$.

It is now immediate to conclude $K=0$.

Q.E.D.

The following is a direct consequence of Theorem 4.1.

Corollary of THEOREM 4.1. Let $M$ be a Sasakian 5-manifold whose sectional curvature satisfies $\tilde{K} \geq \kappa>0$ for a positive constant $\kappa$ and $\Sigma \rightarrow M$ be an orientable, complete open Legendrian surface immersed minimally in $M$.

Suppose that $\Sigma$ satisfies the curvature hypothesis (2).

If the Gaussian curvature $K$ is constant, then $K=0$ or $K$ is a positive constant.

\section{§5. The Simon's type formula for $\Delta_{g}\|\sigma\|^{2}$}

The Simon's type formula can be obtained from a general setting of minimal submanifolds ([5]).

However, for a minimally immersed Legendrian surface fulfilling the curvature hypothesis (2) we observed that the norms satisfy $\|\sigma\|^{2}=2\|\mathscr{Q}\|^{2}$ and $\mathscr{Q}$ is holomorphic.

Therefore, instead of deducing the formula directly we will show the following formula.

LEMma 5.1. If the cubic differential 2 is holomorphic,

$$
\Delta_{g}\|\mathscr{Q}\|^{2}=-2\left\|D^{\prime} \mathscr{Q}\right\|^{2}-6 K\|\mathscr{Q}\|^{2} \text {. }
$$

Here $D^{\prime}$ denotes the $(1,0)$-part of the Hermitian holomorphic connection $D$ on the bundle $\otimes^{3} \mathscr{T}^{*}$, naturally induced from the Levi-Civita connection.

To show this formula we notice that for smooth sections $\mathscr{P}_{l}=\mathscr{P}_{i}(z) d z^{3}$, $i=1,2$ of the bundle $\otimes^{3} \mathscr{T}^{*}$ the Hermitian fibre metric is given 


$$
\left(\mathscr{P}_{1}, \mathscr{P}_{2}\right)(z)=\left(\frac{2}{E}\right)^{3} \mathscr{P}_{1}(z) \overline{\mathscr{P}}_{2}(z) .
$$

Then the connection $D$ is uniquely defined for a complex vector field $X$ in such a way that

$$
X\left(\mathscr{P}_{1}, \mathscr{P}_{2}\right)=\left(D_{X} \mathscr{P}_{1}, \mathscr{P}_{2}\right)+\left(\mathscr{P}_{1}, D_{\bar{X}} \mathscr{P}_{2}\right)
$$

and

$$
D_{\partial / \partial \bar{z}} \mathscr{P}_{1}=\frac{\partial}{\partial \bar{z}} \mathscr{P}_{1}(z) d z^{3}
$$

The $(1,0)$-part of $D$ can be then given

$$
D_{\partial / \partial z} \mathscr{P}_{1}=\left(\frac{\partial}{\partial z} \mathscr{P}_{1}(z)+\beta(z) \mathscr{P}_{1}\right) d z^{3}
$$

where $\beta(z)=-3(\partial / \partial z) \log E$.

Therefore we have in terms of the Gaussian curvature formula (24)

$$
D_{\partial / \partial z}\left(D_{\partial / \partial \bar{z}} \mathscr{P}_{1}\right)-D_{\partial / \partial \bar{z}}\left(D_{\partial / \partial z} \mathscr{P}_{1}\right)=3\left(\frac{\partial^{2}}{\partial z \partial \bar{z}} \log E\right) \mathscr{P}_{1}=-\frac{3}{2} E K \mathscr{P}_{1}
$$

Proof of Lemma 5.1. Since 2 is a holomorphic section of $\otimes^{3} \mathscr{T}^{*}$,

$$
\frac{\partial}{\partial z}(\mathscr{Q}, \mathscr{Q})=\left(D_{\partial / \partial z} \mathscr{Q}, \mathscr{Q}\right)
$$

Thus $\Delta_{g}\|\mathscr{Q}\|^{2}=-(4 / E)\left(\partial^{2} / \partial \bar{z} \partial z\right)(\mathscr{Q}, \mathscr{Q})=-(4 / E)(\partial / \partial \bar{z})\left(D_{\partial / \partial z} \mathscr{Q}, \mathscr{Q}\right)$ is given as

$$
\Delta_{g}\|\mathscr{Q}\|^{2}=-\frac{4}{E}\left\{\left(D_{\partial / \partial \bar{z}} D_{\partial / \partial z} \mathscr{Q}, \mathscr{Q}\right)+\left(D_{\partial / \partial z} \mathscr{Q}, D_{\partial / \partial z} \mathscr{Q}\right)\right\}
$$

which turns out to be the formula of Lemma 5.1, since

$$
D_{\partial / \partial z}\left(D_{\partial / \partial \bar{z}} \mathscr{Q}\right)-\left\{D_{\partial / \partial z}\left(D_{\partial / \partial \bar{z}} \mathscr{Q}\right)-D_{\partial / \partial \bar{z}}\left(D_{\partial / \partial z} \mathscr{Q}\right)\right\}=\frac{3}{2} E K \mathscr{Q} \quad \text { Q.E.D. }
$$

Lemma 5.2. For the holomorphic cubic differential 2

$$
\left\|D^{\prime} \mathscr{Q}\right\|^{2}=\left\|\nabla^{\prime \prime} \sigma\right\|^{2}
$$

where $\nabla^{\prime \prime} \sigma$ denotes the $\xi^{\perp}$-component of $\nabla^{\prime} \sigma$.

Proof. Since the surface is minimal, we can write $\sigma$ as

$$
\sigma=\sigma_{z z} d z^{2}+\bar{\sigma}_{z z} d \bar{z}^{2}
$$

where $\sigma_{z z}=(2 / E) \mathscr{Q}(z) \phi(\partial / \partial \bar{z})$ and then have 


$$
\left(\nabla_{\partial / \partial z}^{\prime \prime} \sigma\right)\left(\frac{\partial}{\partial z}, \frac{\partial}{\partial z}\right)=\frac{2}{E}\left(D_{\partial / \partial z} \mathscr{Q}\right)(z) \phi\left(\frac{\partial}{\partial \bar{z}}\right) .
$$

All other components of $\nabla^{\prime \prime} \sigma$ vanish except for the complex conjugate of (39).

So, $\left\|\nabla^{\prime \prime} \sigma\right\|^{2}=\left\langle\nabla^{\prime \prime} \sigma, \nabla^{\prime \prime} \sigma\right\rangle=(2 / E)^{3} \cdot(E / 2) \cdot\left|(2 / E) D_{\partial / \partial z} \mathscr{Q}\right|^{2}=(2 / E)^{4}\left|D_{\partial / \partial z} \mathscr{Q}\right|^{2}$ $=\left\|D^{\prime} \mathscr{Q}\right\|^{2}$.

Q.E.D.

By applying these lemmata we get the following propositions.

Proposition 5.1. Let $\Sigma \rightarrow M$ be a compact, connected orientable Legendrian surface minimally immersed in a Sasakian 5-manifold $M$.

Suppose $\Sigma$ has nonnegative Gaussian curvature $K$ and satisfies the curvature hypothesis (2).

Then, (i) the immersion $\Sigma \rightarrow M$ is totally geodesic or (ii) $\Sigma$ is flat and $\|\sigma\|$ is constant and the covariant derivative $\nabla^{\prime} \sigma$ has only a $\xi$-component.

Proposition 5.2. Let $\Sigma \rightarrow M$ be a complete, open Legendrian surface immersed minimally in a Sasakian 5-manifold.

Suppose the ambient sectional curvature $\tilde{K}$ of $M$ fulfills $\tilde{K} \leq c$ for a constant $c$.

If $\Sigma$ has $K \geq 0$ and satisfies, same as before, the curvature hypothesis (2), then either $\Sigma \rightarrow M$ is totally geodesic, or $(\Sigma, g)$ is flat and the second fundamental form $\sigma$ has a constant norm and its covariant derivative $\nabla^{\prime} \sigma$ has only a $\xi$-component.

Theorem A, (ii) and Theorem $\mathrm{B}$, (ii) in $\S 1$ are immediate from these propositions.

We will show these propositions.

Proof of Proposition 5.1. First we assume $K=0$ identically. Then from (38) we have

$$
0=\int_{\Sigma} \Delta_{g}\|\mathscr{Q}\|^{2} d \sigma_{\Sigma}=-2 \int_{\Sigma}\left\|D^{\prime} \mathscr{Q}\right\|^{2} d \sigma_{\Sigma}
$$

so $D^{\prime} \mathscr{Q}=0$ and hence from Lemma $5.2 \nabla^{\prime \prime} \sigma$ vanishes.

Now we assume $K$ is not identically zero. Then there is a neighborhood $U$ on which $K>0$. Thus we have

$$
0=-\int_{\Sigma} \Delta_{g}\|\mathscr{Q}\|^{2} d \sigma_{\Sigma} \geq 6 \int_{\Sigma} K\|\mathscr{Q}\|^{2}
$$

Since $K \geq 0, \mathscr{Q}=0$ on $U$ and hence $\mathscr{Q}$ is identically zero from the holomorphicity of 2 . So we get the conclusion.

Q.E.D.

Proof of Proposition 5.2. Take the universal covering surface $\tilde{\Sigma}$ of $\Sigma$. We have then a minimal immersion $\tilde{\Sigma} \rightarrow M$, which is also Legendrian. Since $K \geq 0$, $\tilde{\Sigma}$ is biholomorphic to the complex plane as a Riemann surface. 
From the formula (38) together with $K \geq 0$ it follows $\Delta_{g}\|\mathscr{Q}\|^{2} \leq 0$. So $-\|\mathscr{Q}\|^{2}$ is a subharmonic function which is bounded below, because $-\|\mathscr{Q}\|^{2}=K-$ $\tilde{K} \geq-c$. Over the complex plane any subharmonic function which is bounded below must be constant. Therefore, $\|\mathscr{Q}\|^{2}$ is constant over $\tilde{\Sigma}$ and also over $\Sigma$.

If $\|\mathscr{Q}\|=0$, then, from Lemma 3.1 the immersion $\Sigma \rightarrow M$ is totally geodesic. When $\|\mathscr{Q}\|$ is a positive constant, we have $K=-(1 / 6) \Delta_{g} \log \|\mathscr{Q}\|^{2}=0$ and from Lemmata 5.1 and $5.2 \nabla^{\prime \prime} \sigma=0$.

Q.E.D.

\section{§6. The second variation of minimal immersions}

We will give the second variational formula of area for a minimally immersed Legendrian surface in a Sasakian 5-manifold.

Since the normal bundle $N \Sigma$ splits as $N \Sigma=\phi(T \Sigma) \oplus \boldsymbol{R} \xi$, the space of normal variation vector fields is the direct sum of $\Gamma(\Sigma, \boldsymbol{R} \xi)$ and $\{\phi(V) \mid V \in \Gamma(\Sigma, T \Sigma)\}$.

Proposition 6.1. Let $\Sigma \rightarrow M$ be a compact, connected orientable Legendrian surface minimally immersed in a Sasakian 5-manifold.

Then the index form $\mathscr{I}(\cdot, \cdot)$ associated to the second variation, restricted to $\{\phi(V) \mid V \in \Gamma(\Sigma, T \Sigma)\}$, is given

$$
\begin{gathered}
\mathscr{I}(\phi(V), \phi(W))=\int_{\Sigma}\left\{\left\langle\nabla^{*} \nabla V+(2+K) V, W\right\rangle-\tilde{R} i c_{M}(V, W)\right\} d \sigma_{\Sigma}, \\
V, W \in \Gamma(\Sigma, T \Sigma)
\end{gathered}
$$
$(M, g)$.

Here, $\nabla^{*} \nabla$ denotes the rough Laplacian on $T \Sigma$ and $\tilde{R} i c_{M}$ is the Ricci tensor of

Remark. The Reeb vector field $\xi$ restricted to $\Sigma$ gives a null vector of the index form $\mathscr{I}(\cdot, \cdot)$ so that $\operatorname{nul}(\mathscr{I}) \geq 1$, since $\xi$ generates a 1-parameter isometric contact transformations of $M$.

Proof. The formula of second variation for a minimal immersion is given in [10] as

$$
\mathscr{A}^{\prime \prime}(0)=\int_{\Sigma}\left\{\left\langle\nabla^{\perp} v, \nabla^{\perp} v\right\rangle+\langle\bar{R}(v), v\rangle-\langle\bar{A}(v), v\rangle\right\} d \sigma_{\Sigma}, \quad v \in \Gamma(\Sigma, N \Sigma)
$$

Here (i) $\nabla^{\perp} v$ is the smooth section of $T^{*} \Sigma \otimes N \Sigma$ given by $\left(\nabla^{\perp} v\right)(X)=\nabla_{X}^{\perp} v$ for a tangent vector $X$,

(ii) $\bar{R}, \bar{A}: N_{p} \Sigma \rightarrow N_{p} \Sigma$ are endomorphisms, defined respectively by

$$
\bar{R}(v)=\sum_{i=1}^{2}\left(\tilde{R}\left(e_{i}, v\right) e_{i}\right)^{\perp},
$$

for an orthonormal basis $\left\{e_{1}, e_{2}\right\}$ and

$$
\bar{A}(v)={ }^{t} A(A(v))
$$


for an endomorphism $A: N_{p} \Sigma \rightarrow \otimes^{2} T_{p}^{*} \Sigma,(A(v))(X, Y)=\langle v, \sigma(X, Y)\rangle$ and the adjoint operator ${ }^{t} A$ of $A$.

For a minimal Legendrian surface $\Sigma \rightarrow M$ it is seen that

$$
\langle\bar{A}(v), v\rangle=\|V\|^{2}\|\mathscr{Q}\|^{2}, \quad v=\phi(V) .
$$

We have moreover for $v=\phi(V)$

$$
\langle\bar{R}(v), v\rangle=\sum_{i=1}^{2}\left\langle\tilde{R}\left(e_{i}, \phi(V)\right) e_{i}, \phi(V)\right\rangle .
$$

In order to relate this term to a much more geometrical term we add the following term to both side of the above

$$
\sum_{l=1}^{2}\left\langle\tilde{R}\left(\phi\left(e_{i}\right), \phi(V)\right) \phi\left(e_{i}\right), \phi(V)\right\rangle+\langle\tilde{R}(\xi, \phi(V)) \xi, \phi(V)\rangle
$$

From the $\phi$-invariance of the curvature tensor $\tilde{R}$ together with the equation of Gauss we get the following

$$
\langle\bar{R}(v), v\rangle-\left(K_{p}+\|\mathscr{2}\|^{2}\right)\|V\|^{2}-\|V\|^{2}=-\tilde{R} i c_{M}(V, V),
$$

where we used also the formula $\tilde{R}(X, \xi) X=-\xi$ for a unit vector $X$ orthogonal to $\xi$.

So

$$
\langle\bar{R}(v), v\rangle-\langle\bar{A}(v), v\rangle=-\tilde{R} i c_{M}(V, V)+(1+K)\|V\|^{2}
$$

Since $\nabla^{\perp} v=\phi(\nabla V)+\langle\cdot, V\rangle \xi,\left\langle\nabla^{\perp} v, \nabla^{\perp} v\right\rangle=\langle\nabla V, \nabla V\rangle+\|V\|^{2}$ so that

$$
\int_{\Sigma}\left\langle\nabla^{\perp} v, \nabla^{\perp} v\right\rangle d \sigma=\int_{\Sigma}\left\{\langle\nabla V, \nabla V\rangle+\|V\|^{2}\right\} d \sigma
$$

It follows then

$$
\mathscr{A}^{\prime \prime}(0)=\int_{\Sigma}\left\{\|\nabla V\|^{2}+(2+K)\|V\|^{2}-\tilde{R} i c_{M}(V, V)\right\} d \sigma_{\Sigma}
$$

and the formula in Proposition 6.1 is derived by polarization.

Q.E.D.

Proposition 6.2. For $V \in \Gamma(\Sigma, T \Sigma)$ the index form $\mathscr{I}(\cdot, \cdot)$ can be expressed quadratically

$$
\mathscr{I}(\phi(V), \phi(V))=\int_{\Sigma}\left\{4\|\bar{\partial} \mathscr{V}\|^{2}+2\|V\|^{2}-\tilde{R} i c_{M}(V, V)\right\} d \sigma_{\Sigma}
$$

where we write the dual $V^{\sharp} \in \Gamma\left(\Sigma, T^{*} \Sigma\right)$ of $V$ as $V^{\sharp}=\mathscr{V}+\overline{\mathscr{V}}, \mathscr{V}=\mathscr{V}(z) d z$.

Proof. The above formula is obtained by applying the Weitzenböck formula to $\nabla^{*} \nabla$. 
We have $\left(\nabla^{*} \nabla V\right)^{\sharp}=\nabla^{*} \nabla V^{\sharp}$. So, if we write

$$
\nabla^{*} \nabla V^{\sharp}=\mathscr{V}_{1}+\overline{\mathscr{V}_{1}}
$$

in terms of the $(1,0)$-part $\mathscr{V}_{1}$ together with its complex conjugate, then we have

$$
\mathscr{V}_{1}=\mathscr{V}_{1}(z) d z, \quad \mathscr{V}_{1}(z)=-K \mathscr{V}(z)-\frac{4}{E} \nabla_{\partial / \partial z}\left(\frac{\partial}{\partial \bar{z}} \mathscr{V}(z)\right)
$$

so that

$$
\mathscr{V}_{1}=-K \mathscr{V}+2 \bar{\partial}^{*} \bar{\partial} \mathscr{V}
$$

or

$$
\nabla^{*} \nabla V^{\sharp}=-K V^{\sharp}+2\left\{\bar{\partial}^{*} \bar{\partial} \mathscr{V}+\left(\bar{\partial}^{*} \bar{\partial} \mathscr{V}\right)^{-}\right\} .
$$

Thus, since ${ }^{\sharp}: T \Sigma \rightarrow T^{*} \Sigma$ is isometric,

$$
\begin{aligned}
\left\langle\nabla^{*} \nabla V, V\right\rangle & =-K\left\|V^{\sharp}\right\|^{2}+2\left\langle\bar{\partial}^{*} \bar{\partial} \mathscr{V}+\left(\bar{\partial}^{*} \bar{\partial} \mathscr{V}\right)^{-}, \mathscr{V}+\overline{\mathscr{V}}\right\rangle \\
& =-K\|V\|^{2}+4 \operatorname{Re}\left\langle\bar{\partial}^{*} \bar{\partial} \mathscr{V}, \overline{\mathscr{V}}\right\rangle
\end{aligned}
$$

and by partial integral

$$
\int\left\langle\nabla^{*} \nabla V, V\right\rangle d \sigma_{\Sigma}=\int\left\{-K\|V\|^{2}+4\|\bar{\partial} \mathscr{V}\|^{2}\right\} d \sigma_{\Sigma} .
$$

Hence, we get the desired form of $\mathscr{I}(\cdot, \cdot)$.

Q.E.D.

THEOREM 6.1. Let $\Sigma$ be a compact connected orientable Riemann surface. If $\Sigma \rightarrow M$ be a minimal Legendrian immersion into a Sasakian 5-manifold.

Assume $\left.\tilde{R} i c_{M}\right|_{\Sigma}-2 g>0$ over $\Sigma$. Then

$$
\text { index }(\mathscr{I}) \geq 2 g(\Sigma)
$$

Proof. Notice that the genus $g(\Sigma)$ is given by the dimension of the space of holomorphic 1-forms over $\Sigma$.

For $\mathscr{V} \in H^{0}\left(\Sigma, \mathcal{O}\left(\mathscr{T}^{*}\right)\right)$ we set $V \in \Gamma(\Sigma, T \Sigma)$ by $V^{\sharp}=\mathscr{V}+\overline{\mathscr{V}}$. So from the curvature assumption we get in Proposition 6.2

$$
\mathscr{I}(\phi(V), \phi(V))<0
$$

which shows the required lower bound for the index of $\mathscr{I}(\cdot, \cdot)$.

Q.E.D.

\section{REFERENCES}

[1] D. E. Blair, Contact Manifolds in Riemannian Geometry, Lecture Notes in Math., 509, Sprınger-Verlag, 1976.

[2] D. E. Blair AND K. OGIUE, Geometry of integral submanifolds of a contact distribution, Illino1s J. Math., 19 (1975), 269-276.

[3] W M. Boотhвy AND H. C. WANG, On contact manifolds, Ann. Math., 68 (1958), 721-734. 
[4] B. Y CHEN, Riemannian Submanifolds, 1997, manuscript.

[5] S. S. Chern, M. P do Carmo and S. Kobayashi, Minimal submanifolds of a sphere with second fundamental form of constant length, Functional Analysis and Related Fields (F E. Browder ed.), Springer-Verlag, 1970, 59-71.

[6] Y Hatakeyama, Some notes on differentiable manifolds with almost contact structures, Tôhoku Math. J., 15 (1963), 176-181.

[7] M. KAMEDA, Non-negatively curved $C$-totally real submanifolds in a Sasakıan manifold, Tsukuba J. Math., 2 (1987), 265-272.

[ 8 ] M. J. Micallef and J. G. Wolfson, The second variation of area of minimal surfaces in fourmanifolds, Math. Ann., 295 (1993), 245-267

[9] H. RECKziegel, Horizontal lifts of isometric immersions into the bundle space of a pseudoRiemannian submersion, Lecture Notes in Math., 1156, Springer-Verlag, 264-279.

[10] J. Simons, Minımal varieties in riemannian manifolds, Ann. of Math., 88 (1968), 62-105.

[11] S. Yamaguchi, M. Kon and Y MiYahara, A theorem on $C$-totally real minımal surface, Proc. Amer. Math. Soc., 54 (1976), 276-280.

INSTITUTE OF MATHEMATICS

UNIVERSITY OF TSUKUBA

TSUKUBA, 305-8571, JAPAN

e-mail: Itohm@sakura.cc.tsukuba.ac.jp 